\title{
Preparation of Monodisperse, Fluorescent PMMA-Latex Colloids by Dispersion Polymerization
}

\author{
Gilles Bosma, ${ }^{*}$ Chellapah Pathmamanoharan, ${ }^{*}$ Els H. A. de Hoog, ${ }^{*}$ Willem K. Kegel, ${ }^{*, 1}$ \\ Alfons van Blaaderen, ${ }^{*},{ }^{+}, 2$ and Henk N. W. Lekkerkerker* \\ *Van't Hoff Laboratory for Physical and Colloid Chemistry, Debye Institute, Utrecht University, Padualaan 8, 3584 CH Utrecht, \\ The Netherlands; and $\dagger$ Institute for Atomic and Molecular Physics, Stichting voor Fundamenteel \\ Onderzoek der Materie (FOM), Kruislaan 407, 1098 SJ Amsterdam, The Netherlands
}

Received June 4, 2001; accepted September 20, 2001

\begin{abstract}
We report a single step procedure to prepare monodisperse colloidal poly(methyl)methacrylate (PMMA) particles where fluorescent dyes are incorporated into the polymer network. The particles are sterically stabilized against flocculation by poly(12hydroxystearic acid). The fluorescent dyes are RITC (rhodamine isothiocyanate)-aminostyrene (RAS) and 4-methylaminoethylmethacrylate-7-nitrobenzo-2-oxa-1,3-diazol (NBD-MAEM), which are prepared from (commercially available) RITC and 4-chloro7-nitrobenzo-2-oxa-1,3-diazol (NBD-Cl), respectively, as starting materials. The particles can be grown larger with nonfluorescent PMMA. Examples of the usefulness of these model particles in colloid science are given. (c) 2002 Elsevier Science

Key Words: dispersion polymerization; fluorescent colloids; PMMA-latex; confocal scanning laser microscopy.
\end{abstract}

\section{INTRODUCTION}

Fundamental problems such as phase transitions and dynamics in concentrated systems can very well be studied using colloids that are dispersed into aqueous or apolar solvents $(1,2)$. These systems are used as model systems for (simple) atoms $(3,4)$, but they also are interesting on their own (see, e.g., (5)). Besides that, they are widely used in technological applications such as paints, food processing, drilling fluids, etc.

Recently a number of real-space studies of colloidal systems using confocal scanning laser microscopy (CSLM) have been published (see, e.g., (6-8)). Compared to conventional optical (video) microscopy, the advantage of CSLM is that out-of-focus blur is essentially absent from confocal images, mainly due to a pinhole in front of the detector. This enables one to obtain optical sections from a sample. By using fluorescently labeled particles, interference effects are minimal. Phenomena like crystallization and dynamics are best studied using monodisperse model particles. This places great demands on the model systems. Fluo-

\footnotetext{
${ }^{1}$ To whom correspondence should be addressed. Fax: +31302533870 . E-mail: W.K.Kegel@chem.uu.nl.

${ }^{2}$ Present address: Physics and Chemistry of Condensed Matter, Debye Institute, Princetonplein 5, 3584 CC Utrecht, The Netherlands.
}

rescent particles are not only useful for CSLM studies. Other techniques, such as fluorescent recovery after photobleaching (see for example (9)), also require fluorescent particles.

In this work we describe a method for synthesizing homogeneously labeled fluorescent particles with adjustable size and a sharp size distribution, where fluorescent material is covalently linked to the polymer network of the particle. A simple method for preparing colloidal particles that is widely used in colloid chemistry is a procedure reported by Antl and coworkers (10). In the work presented here, this procedure is modified to incorporate fluorescent monomers into the polymer chains that make up the particles. Two fluorescent monomers are used: NBD-MAEM (4-methylaminoethylmethacrylate-7-nitrobenzo2-oxa-1,3-diazol) and RAS (RITC-aminostyrene), where RITC stands for rhodamineisothiocyanate. A procedure has been published to successfully incorporate the first mentioned monomer into (noncolloidal) poly(methyl)methacrylate (PMMA) (11) and into core-shell colloidal particles (12) using a different procedure (13) from the one used here. The last mentioned monomer has to our knowledge never been incorporated into PMMA.

In the next section we schematically describe how fluorescent particles can be obtained from commercially available starting materials. Subsequently, we describe the experimental details in Section 3, together with the relevant techniques to characterize the particles. In Section 4, the results are given together with a short discussion. Finally we illustrate the usefulness of the newly developed particles as model systems to study a wide variety of phenomena in colloid science.

\section{INCORPORATING FLUORESCENT MONOMERS INTO PMMA-LATEX PARTICLES}

\section{PMMA-Latex Particles}

The latex particles discussed in this paper are referred to as PMMA-latex or polymethylmethacrylate particles and are prepared by dispersion polymerization. In dispersion polymerization the reaction mixture initially consists of monomers that are soluble in the dispersion medium. When the monomers react, polymers are formed that are insoluble in the dispersion medium. 


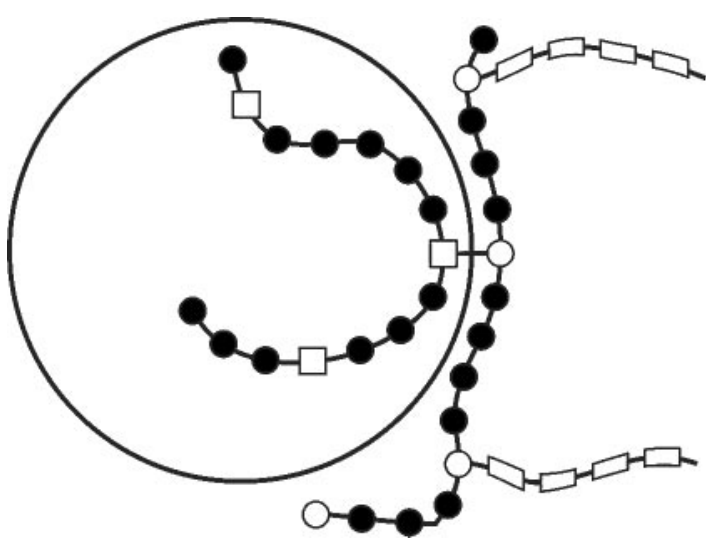

FIG. 1. Schematic representation of a PMMA-latex particle, stabilized by poly(12-hydroxystearic acid) or PHS. Methyl methacrylate (๑); methacrylic acid ( $\square)$; glycidyl methacrylate $(\bigcirc)$; 12-hydroxystearic acid ( $\square$ ).

After a period of time, the polymers precipitate and latex particles are formed $(10,14,15)$. Only apolar media are considered here. The spheres are sterically stabilized by a graft copolymer that coats the latex surface. It is referred to as PHS or poly (12-hydroxystearic acid). A schematic picture of a PMMA sphere is given in Fig. 1.

The monomers methyl methacrylate (MM), methacrylic acid (MA) and glycidyl methacrylate (GM) bind via the double bond of the methacrylic group. In the graft copolymeric stabilizer, to some of the GM monomers, tails of poly(12-hydroxystearic acid) are attached through the ring opening of the epoxy group of the GM and the acid group in the poly(12-hydroxystearic acid). The acid chains originate as a result of esterification between the hydroxy group of a 12-hydroxystearic acid (HS) molecule and the acid group of another HS molecule. The stabilizer is locked onto the latex particle when a GM monomer reacts with a MA monomer at the surface of the PMMA-latex particle.

\section{Fluorescent Monomers}

PMMA-latex can in principle be labeled fluorescently by incorporating dyed monomers homogeneously throughout the particles during the dispersion polymerization. In such a dyed monomer of course both a fluorescent group and a coupling group should be present. In this study we use the monomers 4-methylaminoethylmethacrylate-7-nitrobenzo2-oxa-1,3-diazol (NBD-MAEM) (11) and RITC-aminostyrene (RAS). The coupling group in NBD-MAEM is a methacrylic group. In RAS, the styrene group acts as the coupling group. Because of the chemical equivalence of the coupling groups to the monomers MM and MA, the fluorescent monomers will participate in the polymerization process. Figure 2 shows the reaction scheme that describes the conversion of starting material 4-chloro-7-nitrobenzo-2-oxa-1,3-diazol (NBD-Cl) into NBDMAEM. The reaction product is a fluorescent methacrylate.

The conversion of the dye RITC and 4-aminostyrene into RAS is explained in the reaction scheme of Fig. 3. The reaction product is a fluorescent styrene derivative. From literature it is
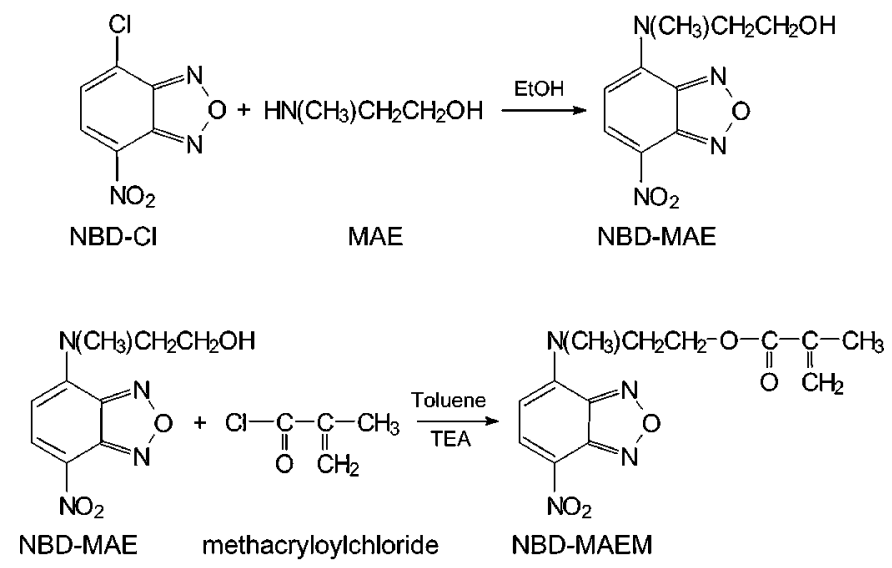

FIG. 2. Reaction scheme of the preparation of NBD-MAEM starting from NBD-Cl.

known that styrene can easily be incorporated into a PMMAlatex matrix $(16,17)$. We note that the methods described above are analogous to the way in which silica is made fluorescent by using FITC or similar dyes (see for example (18)).

The fluorescent monomers NBD-MAEM and RAS can simply be mixed with the nonfluorescent monomer methyl methacrylate. Subsequently, fluorescent PMMA-latex particles are prepared by standard procedures for dispersion polymerization. Details are described in the next section.

\section{EXPERIMENTAL SECTION}

\subsection{Materials}

Methyl methacrylate (MM, BDH, >99\%) was distilled under nitrogen atmosphere before use, methacrylic acid (MA, Fluka, $>98 \%$ ) was distilled at reduced pressure and under nitrogen atmosphere before use. Exxsol D 100 (Exxon Chemical Europe Inc.), hexane (Baker), 40/60 petroleum ether (Baker), octyl mercaptan (Fluka), 2-dimethylaminoethanol (Acros Organics, 99\%), 4-chloro-7-nitrobenzo-2-oxa-1,3-diazol (NBD$\mathrm{Cl}$, Acros Organics, 98\%), methacryloylchloride (Fluka, $\geq 97 \%$ ), 2-methylaminoethanol (MAE, Fluka, $\geq 98 \%$ ), Rhodamine $b$ isothiocyanate (RITC, Fluka, mixture of isomers), 4-aminostyrene (Aldrich), methanol (Baker), diethylether (Merck, $\geq 99.5 \%$ ), and ethanol (Nedalco, absolute technical grade) were used as supplied. Toluene (Baker) was distilled
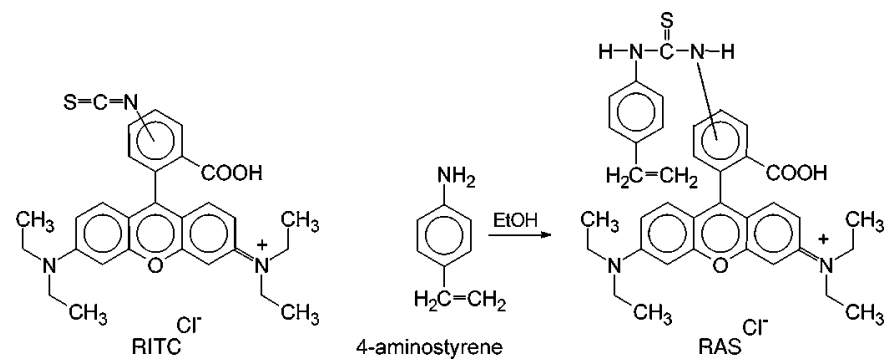

FIG. 3. Reaction scheme of the preparation of RITC-aminostyrene (RAS). 
and dried over Na sand before use. Triethylamine (TEA, Fluka, $\geq 99.5 \%$ ) was distilled and dried over calcium hydride before use. Poly(12-hydroxystearic acid) graft copolymer (50\% solution in a $2: 1(\mathrm{w} / \mathrm{w})$ ethylacetate/butylacetate mixture) was obtained from ICI. Azo-bis-isobutyronitrile (ADIB, Janssen Chimica) was recrystallized from acetone before use.

\subsection{Synthesis of the Fluorescent Monomer NBD-MAEM}

In a Schlenk flask $6.32 \mathrm{~g}$ (84.1 mmol) MAE was dissolved in $32 \mathrm{~mL}$ ethanol. In another Schlenk flask, $2.733 \mathrm{~g}$ (13.7 mmol) NBD-Cl was dissolved in $100 \mathrm{~mL}$ ethanol. The contents of the two flasks were mixed and the mixture was brought under a nitrogen atmosphere. The reaction mixture was stirred and immediately a deposit appeared. An additional amount of $100 \mathrm{~mL}$ ethanol was added. After $30 \mathrm{~min}$ of stirring, the precipitate was filtered with a Büchner funnel.

The residue was washed with a cold $\left(-20^{\circ} \mathrm{C}\right)$ mixture of methanol and diethylether 70/30 (v/v) and then dissolved in $1 \mathrm{~L}$ ethanol of approximately $45^{\circ} \mathrm{C}$. The solution was cooled in the freezer overnight. The NBD-MAE crystals formed were isolated using a Büchner funnel again. The residue was washed with ethanol $\left(T \approx-20^{\circ} \mathrm{C}\right)$. The product was dried in a Schlenk flask under reduced pressure, applied by a membrane pump, at a temperature of $45^{\circ} \mathrm{C}$.

The next step was to convert the NBD-MAE into NBDMAEM. For this purpose, $1.0 \mathrm{~g}(4.20 \mathrm{mmol})$ NBD-MAE was weighed in a Schlenk vessel. The solid was dissolved in $61.3 \mathrm{~mL}$ distilled and dried toluene. The dissolution of the crystals took 3 days. Then, $0.64 \mathrm{~g}(6.3 \mathrm{mmol})$ triethylamine and $0.59 \mathrm{~g}$ (5.6 mmol) methacryloylchloride was added to the solution. The reaction mixture was stirred under nitrogen atmosphere for 5 days. This time span is arbitrary. An ammonium salt precipitated during the course of the reaction. The mixture was filtered, while keeping the system under a nitrogen atmosphere. The reaction product was extracted from the filtrate using a rotating evaporator. The NBD-MAEM was dried for a few hours under reduced pressure.

\subsection{Synthesis of the Fluorescent Monomer RAS}

In a Schlenk flask $0.0712 \mathrm{~g}(0.133 \mathrm{mmol})$ RITC was dissolved in $0.0497 \mathrm{~g}(0.420 \mathrm{mmol}) 4$-aminostyrene and $10.44 \mathrm{~g}$ distilled ethanol. The mixture was brought under a nitrogen atmosphere and was stirred for a period of 4 weeks. We did not follow the conversion in time.

The reaction product was isolated under reduced pressure with a rotating evaporator. The yield of fluorescent monomer was $0.0643 \mathrm{~g}(0.0981 \mathrm{mmol}=73.9 \%)$.

\subsection{Preparation of Fluorescent PMMA-Latex via a Single-Step Synthesis}

The preparation of homogeneous fluorescent latex particles as described in this section was done analogous to the methods described in $(10,19,20)$. For the synthesis a standard reflux setup was built. A 100-mL two-necked round-bottom flask was filled with $0.083 \mathrm{~g}$ ADIB, $9.94 \mathrm{~g}$ MM, $0.205 \mathrm{~g}$ MA, $3.64 \mathrm{~g}$ hexane, $3.04 \mathrm{~g}$ exxsol D 100, $3.29 \mathrm{~g}$ NBD-MAEM/hexane/acetone mixture (consisting of $10 \mathrm{mg}$ NBD-MAEM, $2.64 \mathrm{~g}$ hexane, and $0.65 \mathrm{~g}$ acetone), $1.00 \mathrm{~g}$ stabilizer solution, and $0.054 \mathrm{~g}$ octyl mercaptan. The system was evacuated and brought under nitrogen. The reaction mixture was stirred magnetically. When the ADIB was dissolved completely, the system was heated using an oil bath of $80^{\circ} \mathrm{C}$. A few minutes after the reaction had started, the reaction mixture turned from clear and yellow to a turbid, deep yellow colored solution. The mixture was refluxed for two more hours. The oil bath was removed and the contents of the flask were cooled down to room temperature. The PMMA-latex system that was formed during this reaction was named PMMANBD1. In order to use the latex for further experiments the stabilizer was linked covalently to the particle surface in a second locking distillation step.

For this purpose the dropping funnel was filled with exxsol D 100. The system was heated once more to $80^{\circ} \mathrm{C}$. The hexane was distilled and replaced by the same amount of exxsol D 100. During this process the temperature of the oil bath was slowly raised to $130^{\circ} \mathrm{C}$. When the distillation was complete the distillation condenser was replaced by an allihn condenser. Then $0.86 \mathrm{~g}$ of 2-dimethylaminoethanol was added and the dispersion was refluxed for $2 \mathrm{~h}$. After the locking stage the system was cleaned by removing excess stabilizer and small latex particles through centrifugation of the dispersion. This was done on a Beckman GS-6 table centrifuge for $15 \mathrm{~min}$ at $1000 \mathrm{rpm}$. The sediment was redispersed in 40/60 petroleum ether. The sedimentation procedure was repeated four times. After that, the particles can be transferred to any appropriate solvent (mixture).

Using the same method as described above, 13 more batches of NBD-labeled latex were prepared. Also two batches of rhodamine-labeled latex were synthesized. The only difference between these preparations was that before addition to the reaction mixture, the RITC monomer was dissolved in a mixture of ethanol and hexane.

Details on the amounts of reactants, the radius and polydispersity of the resulting particles can be found in Table 1 .

\subsection{Core-Shell PMMA-Latex}

In (19) a shell of PMMA latex was grown onto (fluorescent) silica cores. We slightly modified this method in order to perform a controlled growth of a PMMA-latex core with a latex shell.

An amount of $10.0 \mathrm{~g}$ PMMA-NBD8 reaction mixture (i.e., the dispersion resulting after synthesis of PMMA-NBD8 particles, see Table 1) was weighted in a 50-mL round-bottom flask. To this solution $0.070 \mathrm{~g}$ ADIB and $0.029 \mathrm{~g}$ octyl mercaptan was added. The mixture was stirred magnetically until the ADIB was dissolved. In a beaker, $3.08 \mathrm{~g} \mathrm{MM}, 0.063 \mathrm{~g} \mathrm{MA}, 2.10 \mathrm{~g}$ hexane, $1.05 \mathrm{~g}$ exxsol D 100, and $0.25 \mathrm{~g}$ stabilizer solution was mixed thoroughly.

The mixture was poured into a dropping funnel with a water jacket, after which the entire system was evacuated and brought 
TABLE 1

Details of the Single-Step Fluorescent Latex Preparations (Amounts in $\mathrm{g})^{a, b}$

\begin{tabular}{|c|c|c|c|c|c|c|c|c|c|}
\hline PMMA-NBD No.: & 1 & $2 \mathrm{~A}$ & $2 \mathrm{~B}$ & 3 & $4 \mathrm{~A}$ & 5 & 6 & 7 & 8 \\
\hline ADIB & 0.083 & 0.105 & 0.104 & 0.107 & 0.102 & 0.102 & 0.100 & 0.099 & 1.045 \\
\hline MM & 9.94 & 10.0 & 10.0 & 10.0 & 10.0 & 10.0 & 10.0 & 10.0 & 100.0 \\
\hline MA & 0.205 & 0.208 & 0.212 & 0.207 & 0.203 & 0.205 & 0.204 & 0.204 & 2.13 \\
\hline Hexane & 3.64 & 6.36 & 6.82 & 6.37 & 5.56 & 5.61 & 5.28 & 3.77 & 68.2 \\
\hline Exxsol D 100 & 3.04 & 4.48 & 4.71 & 4.50 & 4.08 & 4.12 & 3.94 & 4.95 & 47.2 \\
\hline NBD/hexane/acetone mixture & 3.29 & 3.31 & 3.3 & 3.31 & 3.3 & 3.30 & 3.30 & 3.29 & 33 \\
\hline Stabilizer solution (50\%) & 1.00 & 1.02 & 1.07 & 1.02 & 1.07 & 1.02 & 1.02 & 1.00 & 11 \\
\hline Octyl mercaptan & 0.054 & 0.07 & 0.07 & 0.069 & 0.068 & 0.057 & 0.066 & 0.062 & 0.7 \\
\hline 2-Dimethyl aminoethanol & 0.043 & - & - & - & - & - & - & - & - \\
\hline SLS particle radius (nm) & 1880 & 380 & 224 & 295 & 460 & 444 & 615 & 587 & 242 \\
\hline SEM particle radius (nm) & 1849 & 359 & 200 & 278 & 446 & 414 & 581 & 580 & 225 \\
\hline CSLM particle radius (nm) & - & - & - & - & - & - & - & - & 258 \\
\hline SLS polydispersity (\%) & 2.5 & 6.0 & 12.0 & 7.5 & 6.0 & 5.5 & 5.0 & 5.5 & 8.5 \\
\hline \multirow[t]{2}{*}{ SEM polydispersity (\%) } & 3.7 & 5.8 & 6.7 & 4.7 & 2.9 & 4.0 & 2.5 & 3.3 & 6.5 \\
\hline & PMMA-NBD9 & PMMA-NBD10 & \multicolumn{2}{|c|}{ PMMA-NBD11 } & PMMA-NBD12 & PMMA-NBD13 & \multicolumn{2}{|c|}{ PMMA-RITC3 } & PMMA-RITC4 \\
\hline ADIB & 0.1 & 0.09 & \multicolumn{2}{|c|}{0.09} & 0.075 & 0.08 & \multicolumn{2}{|c|}{0.060} & 0.081 \\
\hline MM & 10.0 & 10.0 & \multicolumn{2}{|c|}{5.0} & 5.0 & 5.01 & \multicolumn{2}{|c|}{3.0} & 3.0 \\
\hline MA & 0.204 & 0.205 & \multicolumn{2}{|c|}{0.108} & 0.114 & 0.102 & \multicolumn{2}{|c|}{0.065} & 0.063 \\
\hline Hexane & 4.44 & 3.99 & \multicolumn{2}{|c|}{10.04} & 6.03 & 6.95 & \multicolumn{2}{|c|}{5.46} & 8.77 \\
\hline Exxsol D 100 & 3.52 & 3.3 & \multicolumn{2}{|c|}{5.67} & 3.67 & 4.13 & \multicolumn{2}{|c|}{3.18} & 4.84 \\
\hline NBD/hexane/acetone mixture & 3.3 & 3.3 & \multicolumn{2}{|c|}{1.65} & 1.66 & 1.65 & \multicolumn{2}{|c|}{-} & - \\
\hline RAS/hexane/ethanol mixture & - & - & \multirow{2}{*}{\multicolumn{2}{|c|}{$\overline{0.51}$}} & - & - & \multicolumn{2}{|c|}{$\begin{array}{l}1.80 \\
1.5\end{array}$} & 1.80 \\
\hline Stabilizer solution (50\%) & 1.03 & 1.04 & & & 2.05 & 2.03 & \multirow{2}{*}{\multicolumn{2}{|c|}{$\begin{array}{l}1.51 \\
0.027\end{array}$}} & 1.86 \\
\hline Octyl mercaptan & 0.06 & 0.066 & \multicolumn{2}{|c|}{0.034} & 0.039 & 0.037 & & & 0.027 \\
\hline 2-Diaminoethanol & - & - & \multicolumn{2}{|c|}{-} & 0.046 & 0.045 & \multicolumn{2}{|c|}{0.035} & 0.047 \\
\hline SLS particle radius (nm) & 1000 & 1740 & \multicolumn{2}{|c|}{ Aggr. } & 71 & 43 & \multicolumn{2}{|c|}{-} & - \\
\hline SEM particle radius (nm) & 949 & - & \multirow{2}{*}{\multicolumn{2}{|c|}{ - }} & - & - & \multicolumn{2}{|c|}{95} & 29 \\
\hline CSLM particle radius (nm) & - & - & & & - & - & \multirow{2}{*}{\multicolumn{2}{|c|}{ - }} & - \\
\hline SLS polydispersity (\%) & 3.0 & 3.5 & \multicolumn{2}{|c|}{ - } & - & - & & & - \\
\hline SEM polydispersity (\%) & 1.8 & - & \multicolumn{2}{|c|}{ - } & - & - & \multicolumn{2}{|c|}{15.0} & 13.6 \\
\hline
\end{tabular}

\footnotetext{
${ }^{a}$ Only the batches for which an amount of 2-diaminoethanol is stated have undergone the locking reaction.

${ }^{b}$ A small amount of ethanol was used in order to dissolve the RAS. The ratio in mass of hexane and ethanol in the RAS/hexane/ethanol mixture was $1: 0.98$. The RAS concentration in the particles was approximately $5 \mathrm{mM}$.
}

under a nitrogen atmosphere. The reaction mixture was heated to $80^{\circ} \mathrm{C}$ in an oil bath. The solution in the dropping funnel was added slowly over a period of approximately $30 \mathrm{~min}$. After the complete addition of the monomers the system was refluxed for $2 \mathrm{~h}$. The oil bath was then removed and the dispersion was cooled down to room temperature. The latex particles grown were coded PN8P\#1.

Six more growth reactions were done, using the same method as described above. Five systems (PN8P\#2-PN8P\#6) contained particles with fluorescent cores (PMMA-NBD8) and nonfluorescent shells. The particles of system $\mathrm{P} 2 \mathrm{CN} 1$ were made by the growth of nonfluorescent cores (PMMA-2C) with NBD-labeled shells. The details of these preparations are listed in Table 2.

\subsection{Characterization Techniques}

In order to determine size and polydispersity of the particles, static light scattering (SLS) experiments were performed (21). The measurements were carried out using a Fica-50 photometer as well as a homemade apparatus. Incident and detected light were vertically polarized. The wavelength $\lambda_{0}$ of the incident light was $577 \mathrm{~nm}$. The colloidal systems were made free of dust by filtering them through mitex filters.

Scanning electron microscopy (SEM) was also used to characterize the particles. The dispersions were analyzed on a Philips XL30FEG microscope. Samples were prepared by dipping formfar-coated grids into dilute dispersions. The particles on the grids were coated with a layer of platinum/palladium of $10 \mathrm{~nm}$. A number-averaged particle radius $a$ and its standard deviation $(\sigma)$ were calculated on the basis of the surface area of the spheres. The polydispersity $(\delta)$ of the colloidal systems was defined as $\delta=\sigma / a$.

Samples for CSLM were made by dispersing a drop of the reaction mixture in approximately $5 \mathrm{~mL}$ hexane or petroleum ether. A few drops of the solutions were dried on an object glass. Subsequently the particles were redispersed in a drop of immersion oil and covered with a cover glass. The solutions were very dilute in order to acquire single layers of particles on the object 
TABLE 2

Details of the Growth Reactions (Amounts in $\mathrm{g}$ )

\begin{tabular}{|c|c|c|c|c|c|c|c|}
\hline System & PN8P\#1 & PN8P\#2 & PN8P\#3 & PN8P\#4 & PN8P\#5 & PN8P\#6 & $\mathrm{P} 2 \mathrm{CN} 1$ \\
\hline \multicolumn{8}{|l|}{ Flask } \\
\hline $\begin{array}{l}\text { Reaction mixture } \\
\text { PMMA-NBD8 }\end{array}$ & 10.0 & 10.0 & 10.0 & 5.0 & 5.0 & 3.0 & - \\
\hline $\begin{array}{l}\text { Reaction mixture } \\
\text { PMMA-2C }\end{array}$ & - & - & - & - & - & - & 10.0 \\
\hline ADIB & 0.070 & 0.075 & 0.075 & 0.113 & 0.064 & 0.148 & 0.066 \\
\hline Octyl mercaptan & 0.029 & 0.034 & 0.034 & 0.070 & 0.057 & 0.117 & 0.033 \\
\hline \multicolumn{8}{|l|}{ Dropping funnel } \\
\hline MM & 3.08 & 3.08 & 3.08 & 10.77 & 4.616 & 15.68 & 2.94 \\
\hline MA & 0.063 & 0.063 & 0.063 & 0.220 & 0.094 & 0.32 & 0.060 \\
\hline Hexane & 2.10 & 2.10 & 2.10 & 7.33 & 3.14 & 11.79 & 1.42 \\
\hline Exxsol D 100 & 1.05 & 1.05 & 1.05 & 3.66 & 1.57 & 5.90 & 1.10 \\
\hline NBD/hex./acetone mixture ${ }^{b}$ & - & - & - & - & - & - & 1.00 \\
\hline Stabilizer solution & 0.25 & 0.19 & 0.31 & 0.88 & 0.38 & 1.28 & 0.24 \\
\hline SLS particle radius $(\mathrm{nm})$ & 290 & 292 & 293 & 450 & 365 & (aggr.) & 690 \\
\hline SEM particle radius (nm) & 265 & 266 & 270 & 407 & 336 & - & 637 \\
\hline CSLM particle radius (nm) & 299 & 300 & 306 & 462 & 375 & - & - \\
\hline SLS polydispersity (\%) & 7.5 & 7.5 & 7.5 & 6.0 & 6.0 & - & 4.0 \\
\hline SEM polydispersity (\%) & 7.1 & 6.7 & 5.6 & 7.6 & 7.5 & - & 3.9 \\
\hline
\end{tabular}

${ }^{a}$ This mixture initially consisted of $0.1 \mathrm{~g}$ ADIB, $11.28 \mathrm{~g} \mathrm{MM}, 0.233 \mathrm{~g}$ MA, $7.98 \mathrm{~g}$ hexane, $3.99 \mathrm{~g}$ exxsol, $1.37 \mathrm{~g}$ stabilizer solution (50\%), and $0.06 \mathrm{~g}$ octylmercaptane. The resulting particles were nonfluorescent particles with a radius of $560 \mathrm{~nm}$ and polydispersity as determined by SLS of $6 \%$.

${ }^{b}$ The NBD/hexane/acetone mixture that was used for the growth process was identical to the mixture used in the single step synthesis of fluorescent PMMA-latex particles.

glass. A rough estimate of the mean particle radius resulted from the measurement of the distance of 15 to 20 particles in line.

The systems described in Section 5 are dispersed in various solvents and were sealed in capillaries of $0.1 \mathrm{~mm}$ width, unless stated otherwise.

\section{RESULTS AND DISCUSSION}

\subsection{Single-Step Fluorescent PMMA-Latex}

The radii and the polydispersity of the particles were determined by performing static light scattering (SLS) experiments. Each experimentally obtained scattering pattern was fitted to theoretical formfactors, using Mie theory $(2,21,22)$. The size of the smaller particles (PMMA-NBD12, PMMA-NBD13, PMMARITC3, and PMMA-RITC4) was calculated by doing a Guinier analysis (21). The (SLS and SEM) results under varying conditions are summarized in Table 1.

Figure 4 shows the scattering pattern of latex PMMA-NBD8 (see Table 1). The deep minimum indicates a low polydispersity of the system.

Relative large particles $(a>200 \mathrm{~nm})$ can be made very monodisperse using the single-step method. The trend in these preparations is that the larger the spheres, the lower the polydispersity of the system. Even spheres with a radius as large as $1.9 \mu \mathrm{m}$ could be prepared. The scattering pattern showed $12 \mathrm{~min}-$ ima and a polydispersity of only $2.5 \%$ was found (see Table 1 ).

When plotting the monomer concentrations against the particle radii of the single-step preparations, a nonlinear rela- tion is found. A qualitatively similar result was found by Antl et al. (10). In Fig. 5, a comparison between the preparations of fluorescent-labeled spheres and nonfluorescent spheres is made. The nonfluorescent spheres were synthesized under analogous conditions as described in Section 3. As can be seen in Fig. 5, for identical monomer concentrations, spheres tend to grow larger when fluorescent monomers are incorporated. This is due to the presence of acetone. It makes the reaction mixture more polar and therefore it takes a longer period of time before the growing

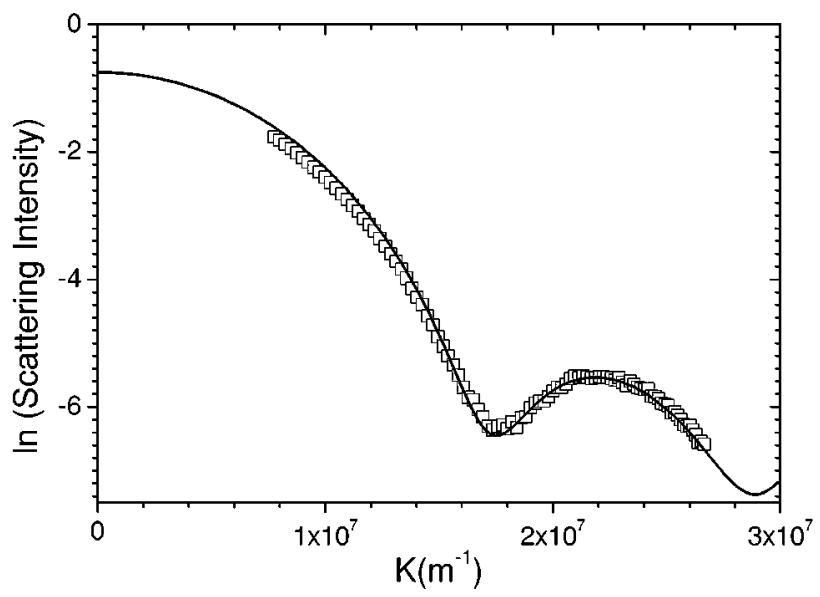

FIG. 4. Experimental (squares) and theoretical (line) scattering pattern of the system PMMA-NBD8 (see Table 1). Mie theory was used to calculate the line, with $242 \mathrm{~nm}$ as particle radius, and $8.5 \%$ as polydispersity. 


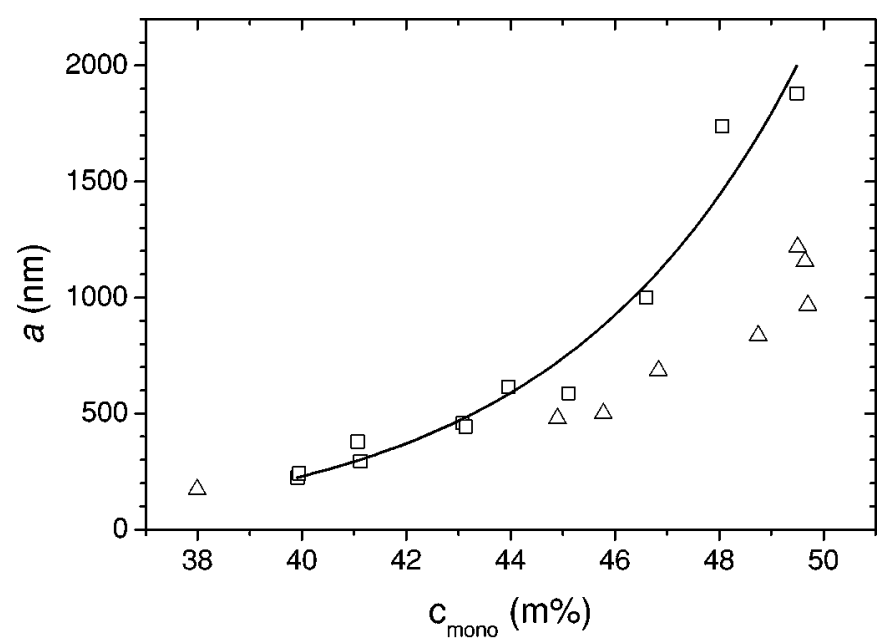

FIG. 5. Particle radii, $a$, of fluorescent and nonfluorescent latices as a function of monomer concentrations, $c_{\text {mono }}$. All systems were prepared using the single-step dispersion polymerization. Open squares correspond to NBD-labeled latices (Table 1), and triangles to nonfluorescent PMMA (not shown in the tables). The line is an exponential fit to the data that correspond to the NBDlabeled latices and is given by $a=-32.6+0.047 \exp \left(0.216 c_{\text {mono }}\right)$.

polymers become unstable. Fewer "nuclei" precipitate and the spheres grow larger. Similarly, it has been found that at higher levels of the polar monomer, larger particles are formed (10). The reason for adding acetone is to dissolve the fluorescent monomer. NBD-MAEM is not soluble in hexane, but it is in acetone.

A convenient method to obtain direct information on particle size and polydispersity is scanning electron microscopy (SEM). In Fig. 6, a SEM photograph of the seed latex PMMA-NBD8 is shown. As the SLS results already suggest, it can be seen from Fig. 6 that the particles are indeed fairly monodisperse. It can also be seen that no secondary latex nucleation took place while preparing the PMMA-NBD8 spheres. This was true for all the single-step latex preparations. It is obvious from Table 1 that both particle size and polydispersity obtained by SEM are

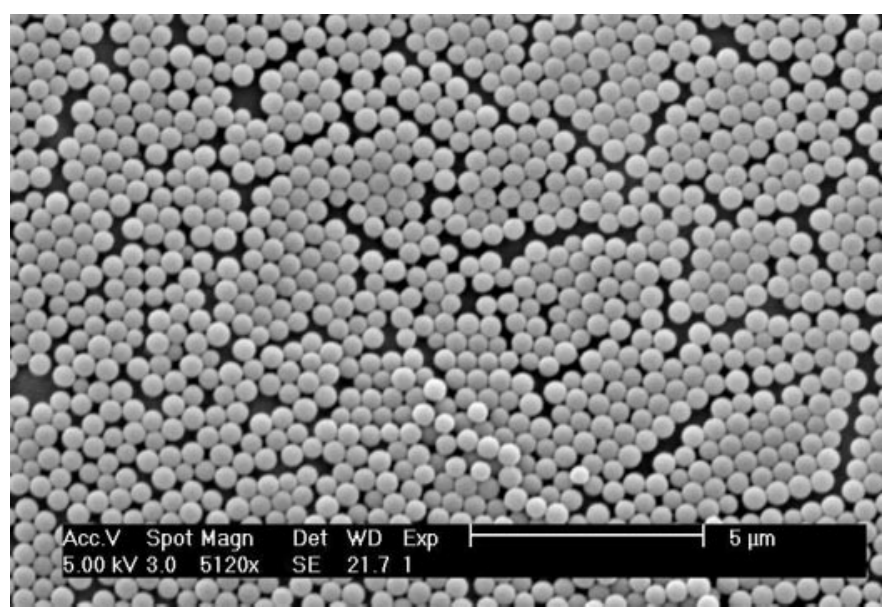

FIG. 6. SEM picture of the system PMMA-NBD8.

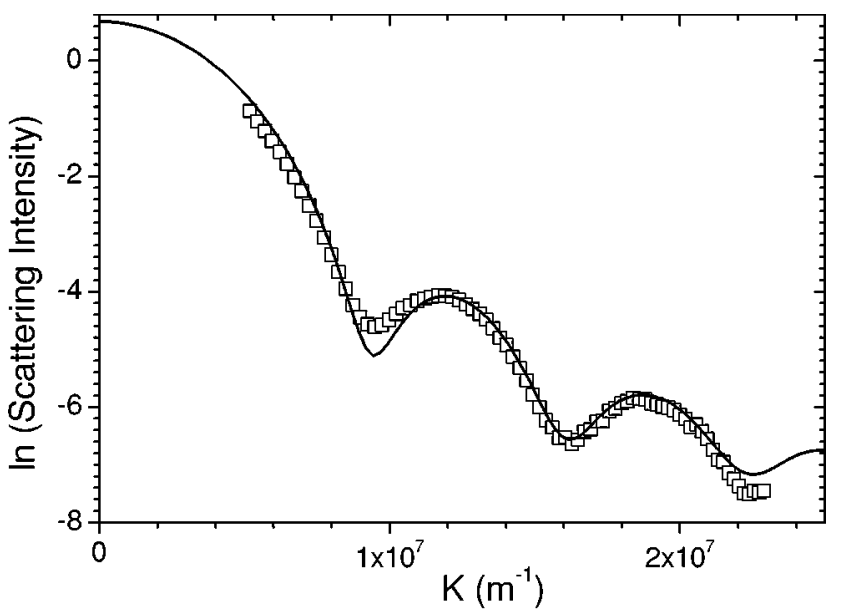

FIG. 7. Experimental (squares) and theoretical (Mie theory) scattering pattern of the system PN8P\#4 in hexane (refractive index 1.375). The theoretical line was calculated using $450 \mathrm{~nm}$ as a particle radius, and $6 \%$ as the polydispersity. The refractive index of the particles is 1.50 and assumed to be homogeneous throughout the particles.

smaller than those obtained by SLS. This is because particles tend to shrink in vacuum (SEM), while multiple scattering and polydispersity have qualitatively similar effects on scattering patterns (SLS). Thus, polydispersity values inferred from SLS are in general too high.

\subsection{Core-Shell PMMA-Latex}

The core-shell systems were also characterized with static light scattering. This was done in the same way as described in the previous section. Small amounts of dye are expected to change the index of refraction of the particles. However, using the refractive index of nonfluorescent PMMA, no significant difference with particle sizes obtained by CSLM was found (see Table 2). Figure 7 shows the scattering pattern of system PN8P\#4. The deep minima again indicate small polydispersity. When comparing Fig. 7 to Fig. 4 one observes a shift of the first minimum to lower scattering vector $(\mathrm{K})$. Furthermore, two additional minima arise, implying that the core/shell particles are larger than the PMMA-NBD8 seeds. It should be noted that the filling up of the minima at low wavevector is mainly due to multiple scattering. At higher wavevector the minima tend to fill up because of the system's polydispersity. A SEM picture of system PN8P\#4 (Fig. 8) shows the formation of small secondary nucleated particles during the growth step.

The larger the amount of PMMA that is grown onto the latex core, the more of these secondary nucleations occur. Adding too many monomers to the seeds leads to irreversible aggregation. This happened when preparing system PN8P\#6. When relatively small amounts of monomer are added, as was done in the preparation of PN8P\#1, PN8P\#2, and PN8P\#3, all the PMMA grows onto the surface of the seed particles. Secondary nucleated PMMA particles can hardly be detected with SLS. The contribution to the scattering intensity of these small particles 


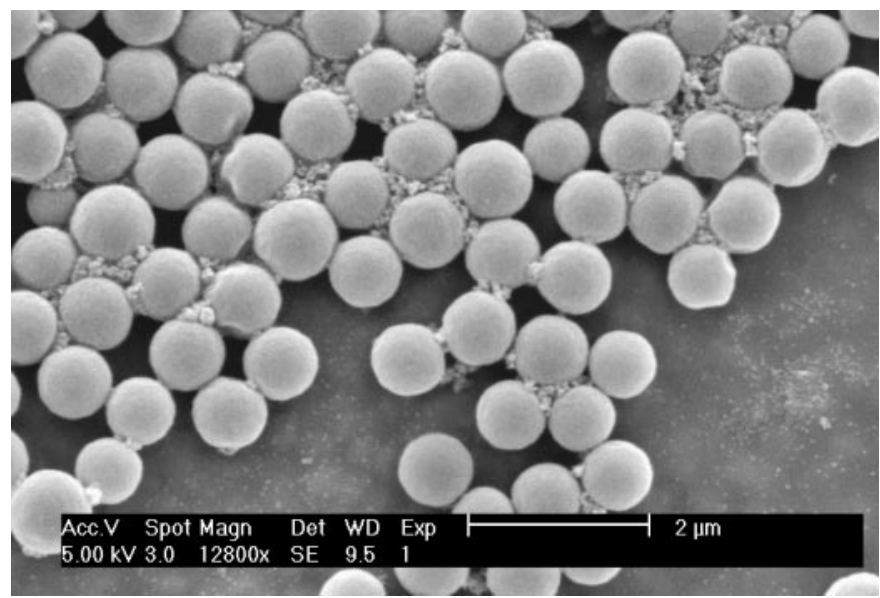

FIG. 8. SEM picture of the system PN8P\#4.

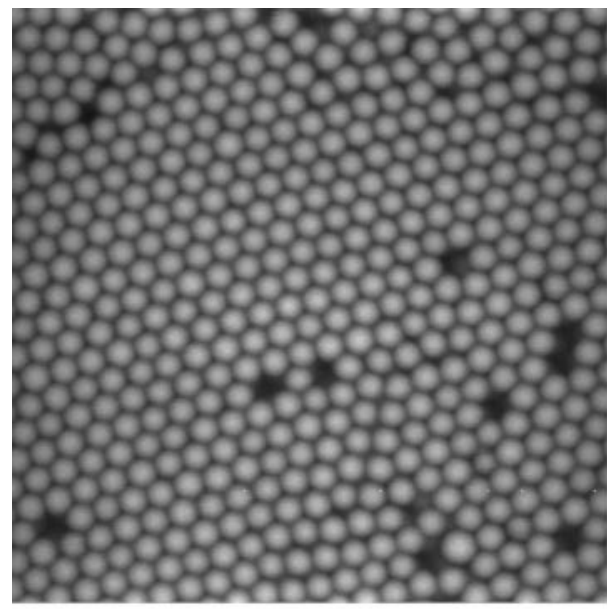

(a) is negligible compared to the contribution of the large spheres. Only at higher wavevector the scattering of the smaller particles becomes noticeable and the minima start to fill up.

When the secondary nucleations are small compared to the core/shell particles, they can easily be removed by centrifugation.

Table 2 lists the experimental details of the growth reactions. The SLS and SEM results are also given.

\subsection{CSLM}

Confocal microscopy was used to verify SLS and SEM results. No extensive quantitative analysis of the pictures was done. Particle radii are based on only 15 to 20 particles. The results are listed in Tables 1 and 2. The CSLM radii are in good agreement with SLS and SEM radii. However, an important conclusion could be drawn after studying the CSLM photographs of the core/shell systems. The thickness of the shells is less than might be expected on basis of the SLS and SEM results. It appears

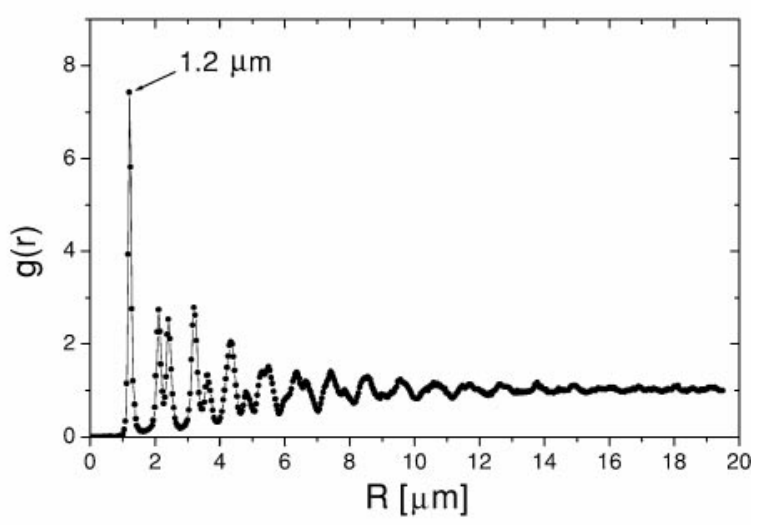

(b)

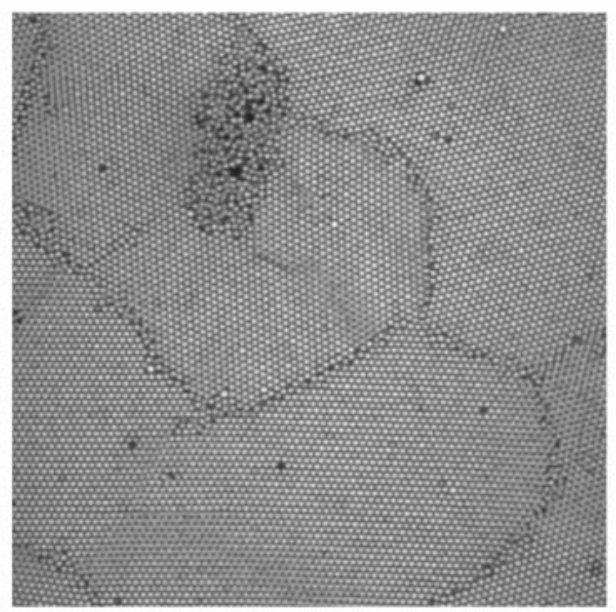

(c)

FIG. 9. (a) NBD-labeled PMMA particles with a diameter of $1.2 \mu \mathrm{m}$ in hexane; (b) $g(r)$ for small PMMA in hexane. Note that the particles behave as hard spheres in this solvent. (c) As (a), but now with smaller magnification in order to visualize boundaries between different colloidal crystallites. 
that the dye is not stationary. In a matter of hours to days, the dye migrates throughout the entire particle and what remains is a system of homogeneous fluorescent spheres. Since the particles do not seem to leak fluorescent material into the solvents (even in solvents where the particles significantly swell up), we believe that fluorescent oligomers are migrating during this process. More work on the mechanism of this process, and ways to avoid it, is currently in progress.

Finally we note that we analyzed the diffraction pattern of a crystallized sample of the system PMMA-NBD\#1 (see Table 1), leading to a particle radius of $2.12 \mu \mathrm{m}$, being in fair agreement with the light scattering and the microscopy results.

\section{APPLICATIONS IN COLLOID SCIENCE}

The newly developed fluorescent PMMA particles are very useful as model systems for studying a variety of phenomena in real space and in real time. As already mentioned in the Introduction, CSLM is a powerful tool for studying the PMMA particles in suspension. In this section we list a number of phenomena that are currently being studied in our groups.

\subsection{Real-Space Structure of Colloidal Crystals}

As shown in Table 1, labeled PMMA particles with diameters ranging from $60 \mathrm{~nm}$ up to at least $4 \mu \mathrm{m}$ can be prepared by our procedures. The polydispersity of all these particles is small. Figure 9a shows a confocal picture of particles with a diameter of $1.2 \mu \mathrm{m}$ in hexane (PMMA-NBD7, see Table 1). Figure 9b shows the radial distribution function, $g(r)$, in two dimensions calculated from the confocal image using image analyses. The first peak in $g(r)$ corresponds to the average positions of the nearest neighbor particles. If the particles behave as hard spheres, this spacing equals the diameter of the particles. From the $g(r)$ we corroborate that the particles indeed have a low polydispersity, as many peaks in $g(r)$ can be distinguished. In Fig. 9c we show the same system but now with smaller magnification in order to visualize boundaries between different colloidal crystallites.

Different solvents may cause different interactions between the particles. Figure 10 shows a confocal image of a sample of large PMMA particles (3.9 $\mu \mathrm{m}$ in diameter) dispersed in a mixture of $96 \%$ of cycloheptyl bromide and $4 \%$ of cis-decalin. An FCC crystal was formed in a very short time. The spacing is 4.7 or $4.86 \mu \mathrm{m}$, depending on which crystal plane is analyzed. As the interparticle spacing is significantly larger than the particle diameter, repulsive interactions between the particles must be present. These repulsive interactions are probably caused by charged groups that are present on the particle surfaces. This charge was also found to be present on particles without dye, so that we may conclude that it is not being caused by the presence of the dye within the PMMA. The mechanism by which charge is induced is unclear at present, but is being studied. Preliminary results suggest that the particles are positively charged.

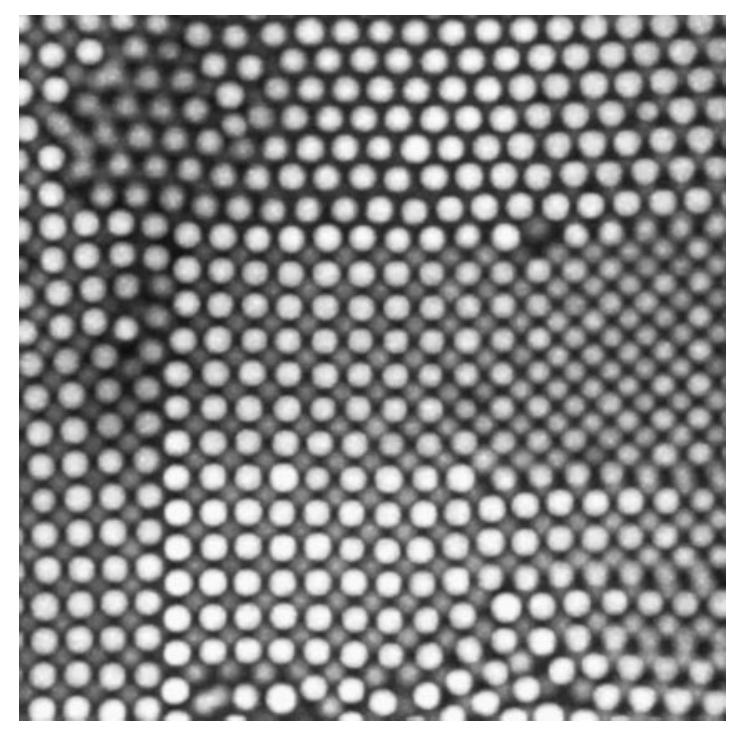

FIG. 10. NBD-labeled PMMA particles with a diameter of $3.9 \mu \mathrm{m}$ in $96 \%$ cycloheptyl bromide. It can be seen that the interparticle distance is significantly larger than the particle diameter.

\subsection{Polymer-Induced Phase Separation}

Dilute colloidal suspensions may separate into a concentrated and a dilute phase in the presence of polymers (23). This process can be followed in time using our newly developed particles in combination with CSLM. In Fig. 11, a confocal image is shown where the growing nuclei of the newly formed phase of colloids with high concentration can clearly be distinguished.

\subsection{Mixtures of NBD and RITC Labeled Particles}

RITC- and NBD-labeled particles can be imaged at the same time, as the emission wavelengths of the two dyes NBD and

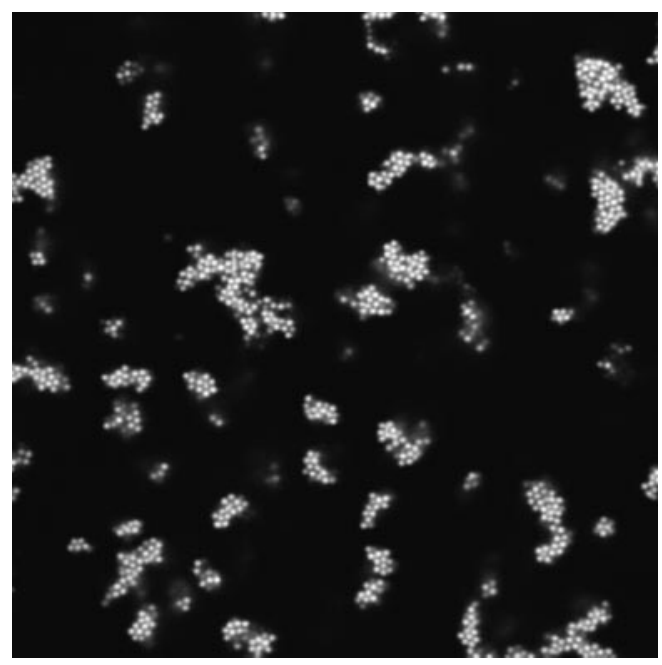

FIG. 11. NBD-labeled PMMA particles with a diameter of $1.2 \mu \mathrm{m}$, and volume fraction of $1.7 \%$, in the presence of polystyrene with a concentration of $2 \mathrm{mg} / \mathrm{mL}$. Image taken $12 \mathrm{~min}$ after mixing colloids with polymer. 


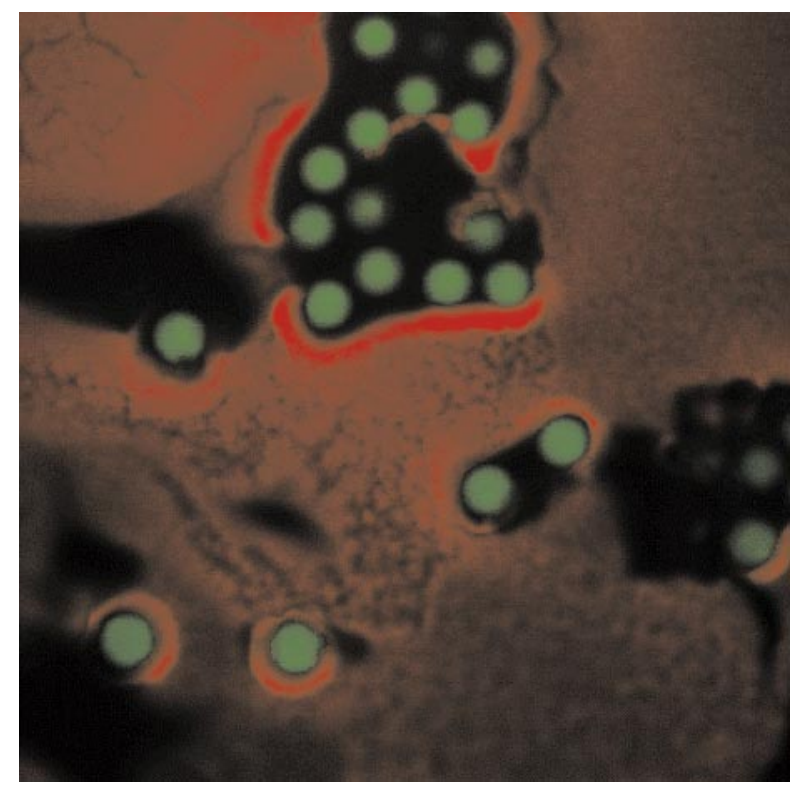

FIG. 12. CSLM pictures of PMMA particles with the two different dyes, RITC (red, particle diameter $60 \mathrm{~nm}$ ) and NBD (green, particle diameter $1.2 \mu \mathrm{m}$ ).

RITC are distinguishable. Figure 12 shows a confocal picture of such mixture: a dried sample of large NBD-labeled particles (PMMA-NBD7; $1.2 \mu \mathrm{m}$ in diameter; as in Fig. 9) and small RITC-labeled PMMA particles (PMMA-RITC4; $60 \mathrm{~nm}$ in diameter). The RITC-labeled particles were too small to be seen separately, but a difference in intensity corresponds to a difference in concentration. So in principle one may study concentration profiles of small particles that are squeezed in between larger particles, or in between walls and larger particles. It appears that RITC bleaches much slower than NBD in this dried sample.

\section{CONCLUSIONS}

We have shown that PMMA-latex particles can be fluorescently labeled by adjusting the single-step dispersion polymerization as described by Antl et al. (10). Small fractions of fluorescent monomers can participate in the polymerization reaction and homogeneous fluorescent particles are obtained. The fluorescent monomers that were used in the experiments were either methacrylate monomers (NBD-MAEM) or styrene monomers (RAS).

SLS and SEM measurements show that the resulting particles are monodisperse and stable against aggregation. Furthermore, secondary nucleations hardly occur during the polymerization process.

The particles can be grown larger by adjusting a growth method similar to the one described in (19). Via a continuous process, particles consisting of a fluorescent core and a nonfluorescent shell of at least $300 \mathrm{~nm}$ thick can be prepared. This growth method leads to stable monodisperse spheres. The sizes of these particles can be tailored. Unfortunately, the dye slowly diffuses throughout the entire particle, thus rendering homogeneous fluorescent spheres. It is at present not clear what the mechanism is of this process.

Using confocal microscopy in combination with numerical techniques, individual particle positions can be resolved so that the newly developed systems are well-suited for studying fundamental processes in colloid science, such as crystallization and diffusion along crystal boundaries. However, as soon as these particles are closer together, on average, than the wavelength of visible light, particle positions cannot be resolved in three dimensions. This will only be possible by using particles with a fluorescent core and a nonfluorescent shell $(6,7)$.

\section{ACKNOWLEDGMENTS}

We thank Roel Dullens and Carlos van Kats for comments on the manuscript.

\section{REFERENCES}

1. Russel, W. B., Saville, D. A., and Schowalter, W. R., "Colloidal Dispersions." Cambridge University Press, Cambridge, 1989.

2. Dhont, J. K. G., An introduction to dynamics of colloids, in "Studies in Interface Science" (D. Möbius and R. Miller, Eds.). Elsevier, Amsterdam, 1996.

3. Pusey, P. N., and van Megen, W., Nature 320, 340 (1986).

4. van Megen, W., and Underwood, S. M., Nature 362, 616 (1993).

5. Larsen, A. E., and Grier, D. G., Nature 385, 230 (1997).

6. van Blaaderen, A., and Wiltzius, P., Science 270, 1177 (1995).

7. Kegel, W. K., and van Blaaderen, A., Science 287, 290 (2000).

8. Weeks, E. R., et al., Science 287, 672 (2000).

9. Imhof, A., et al., J. Chem. Phys. 100, 2170 (1994).

10. Antl, L., et al., Colloid Surf. 17, 67 (1986).

11. Sosnowski, S., Feng, J., and Winnik, M. A., J. Polym. Sci. A 32, 1947 (1994).

12. Kumacheva, E., Kalinina, O., and Lilge, L., Adv. Mater. 11, 231 (1999).

13. O'Callagham, K. Y., Paine, A. Y., and Rudin, A., J. Appl. Polym. Sci. 58, 2047 (1995).

14. Williamson, B., et al., J. Colloid Interface Sci. 119, 559 (1987).

15. Barret, K. E. J., "Dispersion Polymerization in Organic Media." Wiley, London, 1975.

16. Yan, C., Cheng, S. Y., and Feng, L. X., J. Polym. Sci. A 37, 2649 (1999).

17. Kiatkamjornwong, S., et al., Colloids Surf. A 153, 229 (1999).

18. Verhaegh, N. A. M., and van Blaaderen, A., Langmuir 10, 1427 (1994).

19. Bosma, G., et al., submitted for publication.

20. Pathmamanoharan, C., Slob, C., and Lekkerkerker, H. N. W., Colloid Polym. Sci. 267, 448 (1989).

21. Kerker, M., "Scattering of Light and Other Electromagnetic Radiation." Academic Press, New York, 1969.

22. Bohren, G. F., and Huffman, D. R., "Absorption and Scattering of Light by Small Particles." Wiley, New York, 1983.

23. Pusey, P. N., Pirie, A. D., and Poon, W. C. K., Physica A 201, 322 (1993). 